\title{
Pure Laparoscopic Radical Heminephrectomy for a Large Renal-Cell Carcinoma in a Horseshoe Kidney
}

\author{
Rafael B. Rebouças, Rodrigo C. Monteiro, Thiago N. Souza, Paulyana F. Barbosa, George G. Pereira, \\ Cesar A. Britto \\ Urology Department, Edson Ramalho Military Police Hospital, João Pessoa, PB, Brazil
}

Introduction: Horseshoe Kidneys are the most common renal fusion anomaly. When surgery is contemplated for renal-cell carcinoma in such kidneys, aberrant vasculature and isthmusectomy are the major issues to consider. We describe a case of a pure laparoscopic radical heminephrectomy with hand-sewn management of the isthmus for a $11 \mathrm{~cm}$ tumour in a horseshoe kidney.

Presentation: A 47-year-old man complaining of palpable left flank mass for two months. Magnetic resonance of the abdomen revealed a $11 \mathrm{~cm}$ renal mass arising from the left moiety of an incidentally discovered horseshoe kidney. Preoperative CT angiography revealed a dominant anterior renal artery feeding the upper and midpole, with two other arteries feeding the lower pole and isthmus.

The patient was placed in a modified flank position. A four-port transperitoneal technique was used, the colon was reflected. Renal pedicle was dissected and the renal arteries and renal vein were secured with polymer clips. The kidney was fully mobilized and a Satinsky clamp was placed on the isthmus for its division. A running 2-0 vicryl hand-sewn was used for parenchyma hemostasis.

The specimen was extracted intact in a plastic bag through an inguinal incision.

Results: The operative time was 220 minutes, and the estimated blood loss was $200 \mathrm{~mL}$. There were no immediate or delayed complications. The patient resumed oral intake on postoperative day 1 and was discharged on postoperative day 2. Pathologic examination of the specimen confirmed a $11 \mathrm{~cm}$ organ-confined chromophobe renal-cell carcinoma, with negative margins.

Discussion: Laparoscopic oncologic surgery in patients with horseshoe kidneys can be technically challenging. The presence of a large cancer in a horseshoe kidney should not preclude a purely laparoscopic approach. With the aid of a Satinsky clamp, the isthmus can be sharply divided and sutured in a fashion similar to the open technique. To our knowledge, this report represents the largest cancer $(11 \mathrm{~cm})$ removed laparoscopically in the context of a horseshoe kidney.

\section{ARTICLE INFO}

Available at: http://www.brazjurol.com.br/videos/july_august_2013/Reboucas_604_605video.htm

Int Braz J Urol. 2013; 39 (Video \#10): 604-5

Submitted for publication:

February 16, 2013

Accepted after revision:

June 01, 2013

\author{
Correspondence address: \\ Dr. Rafael Batista Rebouças \\ Rua Nossa Senhora dos Navegantes, 188 \\ 508 - Tambaú \\ João Pessoa, PB, 58039-110, Brazil \\ E-mail: rafael.reboucas@gmail.com
}




\section{EDITORIAL COMMENT}

As nicely depicted in this video by Rebouças et al., the surgical management of renal malignancies in the clinical context of aberrant anatomical anomalies such as a horseshoe kidney can be performed in a minimally invasive approach, without adversely impacting cancer specific or overall peri-operative outcomes. The critical factors crucial to optimizing the sequelae of such procedures being an in depth understanding of the location of the tumor with regards to potentially aberrant vascular anatomy as is often frequently exhibited in horseshoe kidneys. In this regard, a pre-operative CT or MRI imaging with 3 (arterial, venous, and delayed) intravascular phases is strongly encouraged, with consideration of a three-dimensional reconstruction of this imaging modality prior to surgery. As in many complex surgical procedures, careful preoperative surgical planning pertaining to anticipated laparoscopic port placement positions, anticipated sequential steps of the surgical procedure, and lastly an appropriate understanding and technical ease with various reconstructive and hemostatic approaches are pivotal. A commitment to each and everyone of all the above steps and principles truly insures a favorable outcome with such minimally invasive complex surgical procedures.

Dr. Philippe E. Spiess Associate Member, Department of Genitourinary Oncology Moffitt Cancer Center, Tampa, FL, USA Video Section Editor, International Brazilian Journal of Urology E-mail: philippe.spiess@moffitt.org 